\title{
VP ellipsis without indices *
}

\author{
Daniel Hardt \\ Copenhagen Business School \\ Nicholas Asher \\ CNRS, IRIT, UMR 5505 \\ Julie Hunter \\ Centre Universitaire Champollion, Albi
}

\begin{abstract}
This paper compares two views on the status of indices in syntactic and logical representations. On a structural view, indices are syntactic formants on a par with node labels and phrase bracketings, and are thus a part of the logical forms that are derived from syntactic representations. On the process view, an index is not a syntactic object at all, but rather, an indication of the output of a resolution process. In this paper we argue that a recent body of data provides a clear empirical basis for distinguishing between these two views of indices. We argue that cases of sloppy VP ellipsis pose insurmountable problems for the structural view of indices, while these problems do not arise for the process view. Furthermore, we show that this resolution process is constrained by the semantics of various discourse relations.
\end{abstract}

Keywords: verb phrase ellipsis, index, dynamic binding, discourse relations

\section{Introduction}

Referential indices have played a central role in many important debates in theoretical linguistics over the past several decades. Their status in syntactic and logical representations, however, is unclear. On one view, indices are syntactic formants on a par with node labels and phrase bracketings, and are thus a part of the logical forms that are derived from syntactic representations. This view, which we term the structural view, is exemplified clearly in Fiengo \& May 1994, and appears to be widely assumed in semantic theories such as CDRT (Muskens 1996). The alternative view, which we will call the process view, holds that an index is not a syntactic object at all, but rather, an indication of the output of a resolution process. This is the approach taken in, for example, SDRT (Asher \& Lascarides 2003).

In this paper, we argue that a recent body of data provides a clear empirical basis for distinguishing between these two views of indices. We argue that cases of sloppy

* Thanks to the audience at SALT 23 for useful and thought-provoking questions. 
VP ellipsis pose insurmountable problems for the structural view of indices, while these problems do not arise for the process view.

\subsection{Background}

An early influential account of sloppy readings in VP ellipsis (VPE) is due to Sag 1976 and Williams 1977. According to this account, a sloppy pronoun must be bound by the local subject, so that both the antecedent and elided VP in (1) are represented as in (2):

(1) Paul thinks he is smart, and Chris does too (think Chris is smart).

(2) $\quad \lambda x . x$ thinks $x$ is smart.

This account cannot handle cases in which a sloppy pronoun varies with an element other than the local subject, as in (3):

(3) First John told Mary I was bad-mouthing her, and then he told SUE I was (bad-mouthing Sue).

To treat such examples, Rooth (1992) loosens the index condition of the SagWilliams account, and adds what might be called a discourse condition: the index condition is that the sloppy variable must be c-commanded by its antecedent; the discourse condition is that there must be appropriate contrast between a constituent containing the ellipisis and some antecedent constituent. This loosened index condition is still too restrictive, as shown by 4; here, the sloppy pronoun is not c-commanded by its antecedent Bill.

(4) The cop who arrested John insulted him. The cop who arrested BILL didn't (insult him).

In response to such examples, Charlow (2012) loosens the index condition further, requiring a relation of dynamic binding between antecedent and sloppy pronoun. Charlow retains Rooth's discourse condition that requires appropriate contrast.

Starting with the Sag-Williams theory, we observe in subsequent approaches a series of looser index conditions, together with reliance on a discourse condition. The proposal of this paper is to remove the index condition entirely, and to rely on a more fine-grained view of discourse conditions. This proposal is motivated largely by data involving sloppy VPE, discovered independently by Hardt (1994) and Schwartz (2000). 
VP ellipsis without indices

\subsection{Sloppy VPE and indexing}

Sloppy VPE is illustrated by (5) with its logical form in (6).

(5) If John has to cook, he doesn't want to (cook). If he has to clean, he doesn't either (want to clean/want to cook).

(6) $\lambda$ C.If John has to C, he doesn't want to C.

Following Hardt 1994, 1999; Schwartz 2000; Merchant 2012; Charlow 2012, we adopt the view that sloppy VPEs should be treated as variables, so that both sentences of (5) are analyzed as in (5/). In this paper we will focus on sloppy VPE examples to argue against a structural account of indexing, and we will propose a process-based approach, in which interpretations arise from the semantics of discourse relations.

The rest of this paper is organized as follows. In section 2, we address the problem of accessibility: we note that this problem is not solved by existing accounts of sloppy VPE, and we argue that this is a serious problem for any structural view of indexing. In section 3, we introduce the first aspect of our account, in which we abandon the structural view, replacing indexed logical forms with underspecified logical forms. In section 4, we review some alternative proposals for sloppy VPE. None of these exploit the semantics of discourse relations, which is the key aspect of the account we propose. In section 5, we argue that the needed constraints are provided by the semantics of various discourse relations and we sketch an account of how different relations constrain VPE and anaphora more generally. Section 6 offers concluding remarks.

\section{The problem: accessibility of VP antecedents}

As both Hardt (1994) and Charlow (2012) point out, variable-based theories of VPE, including the dynamic theories adopted by Hardt and Charlow, face a problem of accessibility. Consider (7):

(7) If John ${ }^{1}$ has to $\operatorname{cook}^{2}$, he $e_{1}$ doesn't $\left[\text { want to } \mathrm{C}_{2}\right]^{3}$. If he ${ }_{1}$ has to clean ${ }^{4}$, he 1 doesn't $C_{3}$ either.

$$
\begin{aligned}
& {\left[u_{1} \mid u_{1}=J o h n\right.} \\
& \quad\left[\mid \text { has-to }\left[C_{2} \mid C_{2}=\lambda u[\mid \operatorname{cook}(u)], C_{2}\left(u_{1}\right)\right]\right] \\
& \quad \Rightarrow\left[C_{3} \mid C_{3}=\lambda u\left[\mid \operatorname{want}\left(u, C_{2}\right)\right], \neg C_{3}\left(u_{1}\right)\right] \\
& \left.\quad\left[\mid \text { has-to }\left[C_{4} \mid C_{4}=\lambda u[\mid \operatorname{clean}(u)], C_{4}\left(u_{1}\right)\right]\right] \Rightarrow\left[\mid \neg C_{3}\left(u_{1}\right)\right]\right]
\end{aligned}
$$

With a straightforward translation into a DRS representation, ${ }^{1}$ the variable $C_{2}$, which represents the first antecedent VP (cook), is embedded in a "box" associated

1 To avoid cluttering the representations, we avoid the nested boxes associated with negations. 
with the modal has to, while $C_{3}$, which represents the second antecedent VP (want to), is embedded in the consequent of the conditional if. The DRS representation is thus ill-formed, because the VPE occurrences represented by $C_{2}$ and $C_{3}$ do not have accessible antecedents.

The accessibility problem is acknowledged without solution by Hardt (1999) and Charlow (2012), though Charlow does suggest that "VP-meanings are 'constant-y' things." Perhaps, therefore, VP meanings could be treated like names in standard DRT, and thus always be accessible on the top level DRS, as shown in (9).

$$
\begin{aligned}
& {\left[u_{1}, C_{2}, C_{3} \mid u_{1}=J o h n,\right.} \\
& \quad \mathbf{C}_{\mathbf{2}}=\lambda \mathbf{u}[\mid \operatorname{cook}(\mathbf{u})], \mathbf{C}_{\mathbf{3}}=\lambda \mathbf{u}\left[\mid \mathbf{w a n t}\left(\mathbf{u}, \mathbf{C}_{2}\right)\right] \\
& \quad\left[\mid \text { has-to }\left[\mid C_{2}\left(u_{1}\right)\right]\right] \Rightarrow\left[\mid \neg C_{3}\left(u_{1}\right)\right] \\
& \left.\quad\left[\mid \text { has-to }\left[C_{4} \mid C_{4}=\lambda u[\mid \operatorname{clean}(u)], C_{4}\left(u_{1}\right)\right]\right] \Rightarrow\left[\mid \neg C_{3}\left(u_{1}\right)\right]\right]
\end{aligned}
$$

Introducing the variables $C_{2}$ and $C_{3}$ in the top level box solves the accessibility problem for the strict reading of (7) (the sloppy reading is discussed later). However, as we shall see, VP meanings cannot always be treated as constants.

\subsection{Non-"constant-y" VPs}

VP meanings are not always constants - some VPs, like the antecedent VP (show her respect) in (10), contain variables.

(10) Whenever a teacher insists that every student has to show her respect, John doesn't want to. And whenever a teacher says that every student has to give her presents, he doesn't either.

How can we solve the accessibility problem with this example? Let's first try representing the VP antecedents in situ:

$$
\begin{aligned}
& {\left[u_{1} \mid u_{1}=J o h n\right.} \\
& {\left[u_{2} \mid \operatorname{teacher}\left(u_{2}\right), \operatorname{insists}\left(u_{2}, \wedge\left[\mid\left[u_{3} \mid \operatorname{student}\left(u_{3}\right)\right] \Rightarrow\right.\right.\right.} \\
& \left.\left.\left.\quad \text { has-to }\left[C_{1} \mid C_{1}=\lambda u\left[\mid \operatorname{show}\left(u, u_{2}, \operatorname{respect}\right)\right], C_{1}\left(u_{3}\right)\right]\right]\right)\right] \\
& \quad \Rightarrow\left[\mathbf{C}_{\mathbf{3}} \mid \mathbf{C}_{\mathbf{3}}=\lambda \mathbf{u}\left[\mid \mathbf{w a n t}\left(\mathbf{u}, \mathbf{C}_{\mathbf{1}}\right)\right], \neg C_{3}\left(u_{1}\right)\right] \\
& {\left[u_{4} \mid \operatorname{teacher}\left(u_{4}\right), \operatorname{says}\left(u_{4}, \wedge\left[\mid\left[u_{5} \mid \operatorname{student}\left(u_{5}\right)\right] \Rightarrow\right.\right.\right.} \\
& \left.\left.\left.\quad \text { has-to }\left[C_{2} \mid C_{2}=\lambda u\left[\mid \operatorname{give}\left(u, u_{4}, \operatorname{presents}\right)\right], C_{2}\left(u_{5}\right)\right]\right]\right)\right] \\
& \left.\quad \Rightarrow\left[\mid \neg C_{3}\left(u_{1}\right)\right]\right]
\end{aligned}
$$

$C_{3}$ is introduced in an embedded box and is therefore inaccessible for the final VPE. 
VP ellipsis without indices

And this time, introducing $C_{3}$ at the top level doesn't solve the problem:

$$
\begin{aligned}
& {\left[u_{1}, C_{3} \mid u_{1}=J o h n, \mathbf{C}_{\mathbf{3}}=\lambda \mathbf{u}\left[\mid \mathbf{w a n t}\left(\mathbf{u}, \mathbf{C}_{\mathbf{1}}\right)\right]\right.} \\
& {\left[u_{2} \mid \text { teacher }\left(u_{2}\right), \operatorname{insists}\left(u_{2}, \wedge\left[\mid\left[u_{3} \mid \operatorname{student}\left(u_{3}\right)\right] \Rightarrow\right.\right.\right.} \\
& \left.\left.\left.\quad \text { has-to }\left[C_{1} \mid C_{1}=\lambda u\left[\mid \operatorname{show}\left(u, u_{2}, \operatorname{respect}\right)\right], C_{1}\left(u_{3}\right)\right]\right]\right)\right] \\
& \quad \Rightarrow\left[\mid \neg C_{3}\left(u_{1}\right)\right] \\
& {\left[u_{4} \mid \operatorname{teacher}\left(u_{4}\right), \operatorname{says}\left(u_{4}, \wedge\left[\mid\left[u_{5} \mid \operatorname{student}\left(u_{5}\right)\right] \Rightarrow\right.\right.\right.} \\
& \left.\left.\left.\quad \text { has-to }\left[C_{2} \mid C_{2}=\lambda u\left[\mid \operatorname{give}\left(u, u_{4}, \operatorname{presents}\right)\right], C_{2}\left(u_{5}\right)\right]\right]\right)\right] \\
& \left.\quad \Rightarrow\left[\mid \neg C_{3}\left(u_{1}\right)\right]\right]
\end{aligned}
$$

While $C_{3}$ is now accessible, the representation is still ill-formed because the variable $C_{1}$ appears free in the definition of $C_{3}$. We could raise $C_{1}$ to the level of $C_{3}$ as in (13), but this would only shift the location of the accessibility problem.

$$
\begin{aligned}
& {\left[u_{1}, C_{1}, C_{3} \mid u_{1}=J o h n,\right.} \\
& \mathbf{C}_{\mathbf{1}}=\lambda \mathbf{u}\left[\mid \mathbf{s h o w}\left(\mathbf{u}, \mathbf{u}_{2}, \operatorname{respect}\right)\right], \mathbf{C}_{\mathbf{3}}=\lambda \mathbf{u}\left[\mid \mathbf{w a n t}\left(\mathbf{u}, \mathbf{C}_{\mathbf{1}}\right)\right] \\
& {\left[u_{2} \mid \operatorname{teacher}\left(u_{2}\right), \operatorname{insists}\left(u_{2}, \wedge\left[\mid\left[u_{3} \mid \operatorname{student}\left(u_{3}\right)\right] \Rightarrow\right.\right.\right.} \\
& \left.\left.\left.\quad \text { has-to }\left[\mid C_{1}\left(u_{3}\right)\right]\right]\right)\right] \\
& \quad \Rightarrow\left[\mid \neg\left(C_{3}\left(u_{1}\right)\right)\right] \\
& {\left[u_{4} \mid \operatorname{teacher}\left(u_{4}\right), \operatorname{says}\left(u_{4}, \wedge\left[\mid\left[u_{5} \mid \operatorname{student}\left(u_{5}\right)\right] \Rightarrow\right.\right.\right.} \\
& \left.\left.\left.\quad \text { has-to }\left[C_{2} \mid C_{2}=\lambda u\left[\mid \operatorname{give}\left(u, u_{4}, \operatorname{presents}\right)\right], C_{2}\left(u_{5}\right)\right]\right]\right)\right] \\
& \left.\quad \Rightarrow\left[\mid \neg C_{3}\left(u_{1}\right)\right]\right]
\end{aligned}
$$

Now $C_{1}$ is accessible, but the variable $u_{2}$ appears free in the definition of $C_{1}$ and moving $u_{2}$ up as well would give the wrong reading. There is no way to move $C_{1}$ high enough to be accessible to the ellipsis occurrence while keeping it low enough to ensure that $u_{2}$ remains bound.

\subsection{Charlow's hybrid approach}

To solve the accessibility problem for variables in VPE representations, Charlow (2012) develops a hybrid account in which a VPE can be represented in two ways.

i. Variable (dynamically-bound)

ii. Deleted (must satisfy Contrast)

We suggest the following representation of (10) for the hybrid approach: 


$$
\begin{aligned}
& {\left[u_{1} \mid u_{1}=J o h n,\right.} \\
& \quad\left[u_{2} \mid \operatorname{teacher}\left(u_{2}\right), \operatorname{insists}\left(u_{2}, \wedge\left[\mid\left[u_{3} \mid \operatorname{student}\left(u_{3}\right)\right] \Rightarrow\right.\right.\right. \\
& \left.\left.\left.\quad \text { has-to }\left[C_{1} \mid C_{1}=\lambda u\left[\mid \operatorname{show}\left(u, u_{2}, \operatorname{respect}\right)\right], C_{1}\left(u_{3}\right)\right]\right]\right)\right] \\
& \quad \Rightarrow\left[\mid \neg \operatorname{want}\left(u_{1}, C_{1}\right)\right] \\
& {\left[u_{4} \mid \operatorname{teacher}\left(u_{4}\right), \operatorname{says}\left(u_{4}, \wedge\left[\mid\left[u_{5} \mid \operatorname{student}\left(u_{5}\right)\right] \Rightarrow\right.\right.\right.} \\
& \left.\left.\left.\quad \text { has-to }\left[C_{2} \mid C_{2}=\lambda u\left[\mid \operatorname{give}\left(u, u_{4}, \operatorname{presents}\right)\right], C_{2}\left(u_{5}\right)\right]\right]\right)\right] \\
& \left.\quad \Rightarrow\left[\mid \neg \text { want }\left(u_{1}, C_{2}\right)\right]\right]
\end{aligned}
$$

The final VPE in (14) is analyzed as a deletion, while the first two are represented as variables, $C_{1}$ and $C_{2}$. (14) does not escape the accessibility problem: $C_{1}$ and $C_{2}$ in the consequent of the top-level conditional are free; their putative antecedents are represented in an embedded context and are thus inaccessible. Nor is the problem solved by introducing $C_{1}$ and $C_{2}$ at the top level-doing so leaves $u_{2}$ and $u_{4}$ free.

$$
\begin{aligned}
& {\left[u_{1}, C_{1}, C_{2} \mid u_{1}=J o h n,\right.} \\
& \mathbf{C}_{\mathbf{1}}=\lambda \mathbf{u}\left[\mid \mathbf{s h o w}\left(\mathbf{u}, \mathbf{u}_{2}, \text { respect }\right)\right] \\
& \mathbf{C}_{\mathbf{2}}=\lambda \mathbf{u}\left[\mid \operatorname{give}\left(\mathbf{u}, \mathbf{u}_{4}, \text { presents }\right)\right] \\
& {\left[u_{2} \mid \operatorname{teacher}\left(u_{2}\right), \operatorname{insists}\left(u_{2}, \wedge\left[\mid\left[u_{3} \mid \operatorname{student}\left(u_{3}\right)\right] \Rightarrow \operatorname{has}-\operatorname{to}\left[\mid C_{1}\left(u_{3}\right)\right]\right]\right)\right]} \\
& \quad \Rightarrow\left[\mid \neg \operatorname{want}\left(u_{1}, C_{1}\right)\right] \\
& {\left[u_{4} \mid \operatorname{teacher}\left(u_{4}\right), \operatorname{says}\left(u_{4}, \wedge\left[\mid\left[u_{5} \mid \operatorname{student}\left(u_{5}\right)\right] \Rightarrow \operatorname{has-to}\left[\mid C_{2}\left(u_{5}\right)\right]\right]\right)\right]} \\
& \left.\quad \Rightarrow\left[\mid \neg \operatorname{want}\left(u_{1}, C_{2}\right)\right]\right]
\end{aligned}
$$

There is an alternative solution available for the hybrid approach that we have not considered, namely, introducing $C_{1}$ and $C_{2}$ at an intermediate level, as in (16):

$$
\begin{aligned}
& {\left[u_{1} \mid u_{1}=\text { John },\right.} \\
& {\left[u_{2}, C_{1} \mid \text { teacher }\left(u_{2}\right), \mathbf{C}_{\mathbf{1}}=\lambda \mathbf{u}\left[\mid \operatorname{show}\left(\mathbf{u}, \mathbf{u}_{2}, \text { respect }\right)\right],\right.} \\
& \left.\quad \text { insists }\left(u_{2}, \wedge\left[\mid\left[u_{3} \mid \operatorname{student}\left(u_{3}\right)\right] \Rightarrow \text { has-to }\left[\mid C_{1}\left(u_{3}\right)\right]\right]\right)\right] \\
& \quad \Rightarrow\left[\mid \neg \text { want }\left(u_{1}, C_{1}\right)\right] \\
& {\left[u_{4}, C_{2} \mid \operatorname{teacher}\left(u_{4}\right), \mathbf{C}_{2}=\lambda \mathbf{u}\left[\mid \operatorname{give}\left(\mathbf{u}, \mathbf{u}_{4}, \text { presents }\right)\right],\right.} \\
& \left.\quad \text { says }\left(u_{4}, \wedge\left[\mid\left[u_{5} \mid \operatorname{student}\left(u_{5}\right)\right] \Rightarrow \operatorname{has}-\operatorname{to}\left[\mid C_{2}\left(u_{5}\right)\right]\right]\right)\right] \\
& \left.\quad \Rightarrow\left[\mid \neg \text { want }\left(u_{1}, C_{2}\right)\right]\right]
\end{aligned}
$$

In Charlow's hybrid approach, both VPE as a variable and VPE as deletion can be viewed as generating a presupposition that there is an accessible antecedent. For VPE as deletion, the VPE must be contained in a constituent under the scope of Rooth's squiggle operator $(\sim)$, so $\alpha \sim$ presupposes a $\beta$ such that $\beta \in\langle\langle\alpha\rangle$, where $\langle\langle\alpha\rangle\rangle$ is the focus-value of $\alpha$. For VPE as a variable, there is simply a presupposition of an accessible coindexed antecedent $\alpha$. In either case, because a VPE occurrence generates a presupposition of an accessible antecedent, a non-accessible antecedent 
VP ellipsis without indices

will trigger accommodation. Following the Trapping constraint of van der Sandt 1992, the presupposition must appear at a level where it is accessible to the VPE, and when it is accommodated, the most global possible accommodation is preferred.

The hybrid approach may be able to solve the version of the accessibility problem posed by (10) by appealing to intermediate accommodation. However, we see no independent motivation for positing these two different approaches to VPE. Our approach provides a uniform analysis of VPE as variables; it removes indexing constraints, and relies instead on the semantics of discourse relations. In our view, this is simpler and more well-motivated. Furthermore, as we show below, relying on discourse relations makes it possible to capture a range of observations that have not been accounted for in other approaches.

\section{Proposal part 1: no index constraints}

We propose removing all indexing constraints for VPE (and for anaphoric expressions in general). Instead, we will exploit underspecified representations of the kind used in SDRT (Asher \& Lascarides 2003). According to our view, anaphoric expressions, including VPE, introduce 'holes' or underspecified elements into logical form, which we represent with question marks, as shown in (18), the logical form for (10) repeated below.

(17) Whenever a teacher insists that every student has to show her respect, John doesn't want to. And whenever a teacher says that every student has to give her presents, he doesn't either.

$$
\begin{aligned}
& {\left[u_{1}, C_{1}, C_{2}, C_{3} \mid u_{1}=J o h n,\right.} \\
& C_{1}=\lambda u[z \mid \operatorname{show}(u, z, \text { respect }), z=?], \\
& C_{2}=\lambda u\left[z_{1} \mid \operatorname{give}\left(u, z_{1}, \text { presents }\right), z_{1}=?\right], \\
& C_{3}=\lambda u\left[C_{4} \mid \operatorname{wants}\left(u, C_{4}(u)\right), C_{4}=?\right], \\
& {\left[u_{2} \mid \operatorname{teacher}\left(u_{2}\right), \operatorname{insists}\left(u_{2}, \wedge\left[\mid\left[u_{3} \mid \operatorname{student}\left(u_{3}\right)\right] \Rightarrow \operatorname{has}-\operatorname{to}\left[\mid C_{1}\left(u_{3}\right)\right]\right]\right)\right]} \\
& \quad \Rightarrow\left[\mid \neg \operatorname{want}\left(u_{1}, C_{5}\right), C_{5}=?\right], \\
& {\left[u_{4} \mid \operatorname{teacher}\left(u_{4}\right), \operatorname{says}\left(u_{4}, \wedge\left[\mid\left[u_{5} \mid \operatorname{student}\left(u_{5}\right)\right] \Rightarrow \operatorname{has}-\operatorname{to}\left[\mid C_{2}\left(u_{5}\right)\right]\right]\right)\right]} \\
& \left.\quad \Rightarrow\left[C_{6} \mid \neg C_{6}\left(u_{1}\right), C_{6}=?\right]\right]
\end{aligned}
$$

(18) has a set of interpretations, one for each way of filling in possible values for the underspecified elements, where the possible values are provided by the accessible discourse referents in the context. At the model theoretic and dynamic semantic level, an underspecified formula of the form $z=$ ? is a test (like other formulas in a DRS's condition list) that passes all world assignment pairs where $z$ is assigned some 
value in the input assignment. Such a formula does not contribute semantic content until its interpretation is filled in and the ? is replaced by an accessible discourse referent. ${ }^{2}$ We discuss the role of discourse relations in filling in an interpretation in section 5. In the meantime, (19) provides an example interpretation of (18), namely, that of the preferred sloppy reading:

$$
\begin{aligned}
& {\left[u_{1}, C_{1}, C_{2}, C_{3} \mid u_{1}=J o h n,\right.} \\
& C_{1}=\lambda u[z \mid \operatorname{show}(u, z, \text { respect }), z=?] \text {, } \\
& C_{2}=\lambda u\left[z_{1} \mid \operatorname{give}\left(u, z_{1} \text {, presents }\right), z_{1}=\right.\text { ?], } \\
& C_{3}=\lambda u\left[C_{4} \mid \operatorname{wants}\left(u, C_{4}(u)\right), C_{4}=\text { ? }\right] \text {, } \\
& {\left[u_{2} \mid \operatorname{teacher}\left(u_{2}\right), \operatorname{insists}\left(u_{2}, \wedge\left[\mid\left[u_{3} \mid \operatorname{student}\left(u_{3}\right)\right] \Rightarrow \operatorname{has}-\operatorname{to}\left[\mid C_{1}^{z \rightarrow u_{2}}\left(u_{3}\right)\right]\right]\right)\right]} \\
& \Rightarrow\left[\mid \neg C_{3}^{C_{4} \rightarrow C_{1}^{z_{1} \rightarrow u_{2}}}\left(u_{1}\right)\right] \\
& {\left[u_{4} \mid \operatorname{teacher}\left(u_{4}\right), \operatorname{says}\left(u_{4}, \wedge\left[\mid\left[u_{5} \mid \operatorname{student}\left(u_{5}\right)\right] \Rightarrow \operatorname{has}-\operatorname{to}\left[\mid C_{2}^{z_{1} \rightarrow u_{4}}\left(u_{5}\right)\right]\right]\right)\right]} \\
& \left.\Rightarrow\left[C_{6} \mid \neg C_{6}\left(u_{1}\right), C_{6}=C_{3}^{C_{4} \rightarrow C_{2}^{z_{1} \rightarrow u_{4}}}\right]\right]
\end{aligned}
$$

The superscripts on the property discourse referents in (19) indicate how underspecified elements in their descriptions are to be resolved in context. Note, however, that the question marks in the descriptions remain in the final representation. This reflects both the fact that an interpreter might revise his original interpretation of a VPE occurrence in light of new information, as well as the fact that the properties in a VPE occurrence can be picked up again and reused as antecedents as the discourse proceeds. Imagine, for example, continuing (10) with 'But whenever a teacher asks that every student bring her chocolate, he does!'. For readability, though, we can ignore the update potential of the entries by eliminating the question marks and equations and directly plugging in the values given by the superscripts in (19).

$$
\begin{aligned}
& {\left[u_{1}, \mid u_{1}=J o h n,\right.} \\
& {\left[u_{2} \mid \operatorname{teacher}\left(u_{2}\right),\right.} \\
& \left.\quad \text { insists }\left(u_{2}, \wedge\left[\mid\left[u_{3} \mid \operatorname{student}\left(u_{3}\right)\right] \Rightarrow \text { has-to }\left[\mid \operatorname{show}\left(u_{3}, u_{2}, \text { respect }\right)\right]\right]\right)\right] \\
& \Rightarrow\left[\mid \neg \operatorname{want}\left(u_{1}, \operatorname{show}\left(u_{1}, u_{2}, \operatorname{respect}\right)\right)\right] \\
& {\left[u_{4} \mid \operatorname{teacher}\left(u_{4}\right),\right.} \\
& \quad \text { says }\left(u_{4}, \wedge\left[\mid\left[u_{5} \mid \operatorname{student}\left(u_{5}\right)\right] \Rightarrow \text { has-to }\left[\mid \operatorname{give}\left(u_{5}, u_{4}, \operatorname{presents}\right)\right]\right.\right. \\
& \left.\quad \Rightarrow\left[\mid \neg \operatorname{want}\left(u_{1}, \operatorname{give}\left(u_{1}, u_{4}, \operatorname{presents}\right)\right)\right]\right]
\end{aligned}
$$

2 Or alternatively until a specific value for $z$ in the input assignment is selected, as in continuation style representations of anaphoric expressions (de Groote 2006). 
VP ellipsis without indices

\section{Alternative solutions}

\subsection{Freely resolve VPE}

Several accounts have been proposed for examples of sloppy VPE like (5), repeated here:

(21) If John has to cook, he doesn't want to. (cook).

If he has to clean, he doesn't either. (want to clean/want to cook)

Tomioka (2008) offers a syntactic deletion account of VPE according to which deletion is determined in a "step-by-step" fashion. As Tomioka points out, sloppy VP examples like (5) contradict the straightforward version of a syntactic deletion account, which holds that a VP can be elided only if it is identical with some overt VP. On the sloppy reading of (5), the VP want to clean is elided even though it is not identical to any other VP. In Tamioka's account, the VP want to clean can be deleted because "the content of what has already been decided to be deleted should not play a role" (219). As an illustration, the analysis of (5) proceeds as follows:

\section{want to clean step 1: delete clean}

want to $<>\quad$ step 2: delete want to $<>$

First, clean is deleted by identity with the previous occurrence of clean. This results in want to $\langle>$. At this point, the deleted portion can be ignored, and because of that, the previous occurrence of want to $<>$ licenses deletion. Merchant (2012) develops a similar proposal based on syntactic deletion, though he argues that there is no need for a derivational view like that of Tamioka's. Merchant proposes instead a special criterion of $e$-giveness for ellipsis, which has a similar effect to Tamioka's step by step procedure.

- e-givenness: An expression $X$ is $e$-given iff $X$ has a salient antecedent $A$ and, modulo existential type-shifting,

i. $A$ entails $\mathrm{E}-\operatorname{clo}(X)$, and

ii. $X$ entails $\mathrm{E}-\operatorname{clo}(A)$, where $\mathrm{E}-\operatorname{clo}(\alpha)$ is the result of replacing all $\mathrm{E}$ marked sub-elements of $\alpha$ with existentially bound variables of the appropriate type,

A constituent $\alpha$ can be elided if $\alpha$ is e-given. The operation of E-closure produces the following representation for the elided (5):

- $\exists x . \exists \phi . x \neg$ wants to $\phi$ 
In effect, E-closure allows elided sub-elements to be ignored in licensing ellipsis.

Both Merchant and Tomioka show that sloppy VPE is possible within a syntactic deletion account by stipulating that differences in elided sub-elements can be ignored. Without this stipulation, the syntactic deletion account is too restrictive to permit sloppy VPE. Unfortunately, this stipulation renders a deletion account too permissive because it does not capture the need for parallel controllers, which, we will argue, are an essential feature of sloppy readings.

\subsection{The need for parallel controllers}

A crucial point emphasized in work that introduces the phenomenon of sloppy VPE is that the antecedents in an example like (5) are parallel controllers (see Hardt 1994, 1999, inter alia.). Just as sloppy pronouns require parallel controlling NPs, sloppy VPEs require parallel controlling VPs. Yet nothing is said about this constraint in Tomioka's or Merchant's account of sloppy VPE. These accounts would therefore incorrectly permit ellipsis in the absence of parallel controllers, as illustrated by (22):

(22) I often wanted John to help me with my writing. When John had to clean, he didn't want to. When he had to cook, he didn't either.

Ok Readings:

Strict1: help me/help me

Strict2: clean/clean

Sloppy: clean/cook

\section{Bad Readings:}

help me/help me

clean/clean

clean/cook

Merchant's and Tomioka's accounts would permit all of the bad readings along with the good ones. Similar observations can be made with pronouns:

(23) Sometimes Sam needs help when other students cause trouble - but I only step in when needed. If John was making trouble in class I would help him, but if Bill was making trouble I wouldn't.

\section{Ok Readings:}

Strict1: help Sam/help Sam

Strict2: help John/help John

Sloppy: help John/help Bill

\section{Bad Readings:}

help Sam/help Bill

help Sam/help John

help John/help Sam

The challenge, then, is to capture the flexibility required to allow sloppy readings, while incorporating a constraint involving parallel controllers. As Schwartz (2000) points out, this challenge can be met if the sloppy expression is bound by the controller. Given the possibility for binding, the sloppy readings are correctly 
VP ellipsis without indices

treated on a par with the strict reading or readings, and distinguished from the other switching readings. Neither Merchant nor Tomioka propose any binding mechanism, which is why their accounts are not able to rule out the bad readings.

\subsection{Structure sharing}

Sauerland (2007) proposes an alternative solution, based on focus dependencies with structure sharing. On this approach, the sloppy expression shares syntactic structure with the controller, and thus co-varies with the controller in the computation of focus alternatives. Thus in (22) the focused occurrence of cook and the elided occurrence of cook share the same syntactic structure. This means that the focus alternatives are of the form, When he had to X, he didn't want to X either. This avoids the problem of the bad readings described above. Below we will describe a different approach, where the parallel controllers requirement arises from the semantics of relevant discourse relations such as Parallel and Contrast. In our view, this is attractive in that the semantics of discourse relations is independently motivated, as discussed at length in the literature on discourse and semantics (see for example Asher 1993; Asher \& Lascarides 2003 for relevant overview and references). Whether Sauerland's notion of syntactic structure sharing has independent motivation is a matter we will leave to the syntacticians. We will point out a potential challenge for this approach. It is well known that ellipsis occurrences often differ syntactically from their antecedents. In what follows, the examples in (24) show a difference of number and the examples in (25) show a difference in polarity.

(24) When teachers demand that students give them their addresses, John doesn't want to. And when teachers demand that students give them their phone numbers, John doesn't either.

Here, Sauerland's account should produce the focus value When teachers demand that students $X$, John doesn't want to $X$, and this is explained by positing syntactic structure sharing between the two occurrences of X. However, it would appear that the two occurrences of $X$ are syntactically distinct, since the first occurrence is give them their phone numbers, and the second is give them his phone number.

(25) When the teacher says you shouldn't do any reading, John wants to. And when the teacher says you shouldn't solve any problems, John does too.

Again, Sauerland's account requires structure sharing between the antecedent solve any problems and the elided VP, which is solve some problems. The question 
is whether there is an independently motivated notion of syntactic structure sharing that would be flexible enough to permit deviations of this sort.

In the next section, we show how discourse relations can provide both the flexibility and constraints that are required for these examples.

\section{Proposal part 2: discourse constraints}

\subsection{Overall architecture}

In section 3, we introduced a compositional mechanism for constructing underspecified DRSs for VPE. We turn now to the second aspect of our account, that of explaining the process by which the preferences and constraints imposed by certain discourse relations serve to order and filter possible interpretations of our underspecified logical forms.

\subsection{Parallel relations and Maximal Common Theme}

In many examples of ellipsis, the constituent containing the ellipsis is linked to the constituent containing the antecedent by either the PARALLEL relation or the CONTRAST $^{3}$ relation. ${ }^{4}$ For instance, the two sentences involved in both (5) and (10) are linked by PARALLEL, which is signaled by the presence of either.

Discourse relations impose constraints on the resolution of ellipsis and other anaphors. Following Asher, Hardt \& Busquets 2001, PARALLEL imposes a constraint on the two discourse units that it relates, which involves checking that the two units have a common structure and lexical content - this is one way of analyzing the idea of parallel controllers. To make this precise, we appeal to the notion of a maximal common theme (MCT). While a theme can always be constructed between any two discourse units, different themes may exhibit varying degrees of abstraction from the content of the units from which they are created. We construct a common theme from a DRS $\alpha$ by applying a sequence of operations, which we term generalizations, as follows:

\section{Definition 1 Generalizations:}

- Delete a term

- Rename a variable binder

3 We use small caps here rather than bold face to indicate the significant difference between Rooth's notion of contrast and ours.

4 In fact, Asher (1993) argues that ellipsis is an important clue for the presence of PARALLEL and CONTRAST; that is, an ellipsis disposes speakers to interpret the discourse unit containing the ellipsis as linked to the discourse context by one of these relations. 
VP ellipsis without indices

- Lambda abstract over one or more identical elements

Definition 2 For DRS's $\alpha$ and $\beta$, a common theme $C T$ is one that results from applying a sequence of generalizations $g_{1} \circ g_{2} \circ \ldots \circ g_{n}=\sigma$ to both $\alpha$ and $\beta$. We write this $C T=\sigma(\alpha)=\sigma(\beta)$.

Themes or common themes thus have a natural ordering $>$.

Definition $3 C T>C T^{\prime}$ iff $\exists \sigma \sigma(C T)=C T^{\prime} \wedge \neg \exists \sigma^{\prime} \sigma^{\prime}\left(C T^{\prime}\right)=C T$

A common theme $C T$ of $\alpha$ and $\beta$ is a maximal common theme if there is no common theme $C T^{\prime}$ of $\alpha$ and $\beta$ such that $C T^{\prime}>C T$. While we use the terminology of Asher et al. 2001, other people have had similar ideas (Prust, Scha \& van den Berg 1994; Asher 1993; Dalrymple, Sheiber \& Pereira 1991).

The relation PARALLEL requires that its constituents be interpreted such that they result in a maximal common theme. More specifically, consider two underspecified DRS's $\alpha$ and $\beta$, related by PARALLEL:

- For each fully specified $\alpha_{i}$ and $\beta_{i}$, determine $\operatorname{MCT}\left(\alpha_{i}, \beta_{i}\right)$

- Consider any two fully specified pairs $\left.<\alpha_{i}, \beta_{i}\right\rangle,\left\langle\alpha_{j}, \beta_{j}>\right.$, with corresponding maximal common themes, $M C T_{i}$ and $M C T_{j}$

- If $M C T_{i}>M C T_{j}$, the interpretation associated with $<\alpha_{i}, \beta_{i}>$ is preferred

\subsubsection{Applications of maximal common theme}

To illustrate, let's return to example (22) repeated below:

(26) I often wanted John to help me with my writing. When John had to clean, he didn't want to. When he had to cook, he didn't either.

(22) has a preferred strict reading in which the ellipses are replaced with help me with my writing and want to help me with my writing. There is also an acceptable sloppy reading, in which we replace the first ellipsis with clean and the second with want to cook, which is helped by inserting an and at the beginning of the second sentence. There is also a bad sloppy reading where the the first ellipsis is resolved as help me with my writing and the second is resolved as want to cook. Consider first the acceptable sloppy reading, shown in (27). The two relevant DRSs are labeled $\alpha$ and $\beta$ and related by PARALLEL.

$$
\begin{aligned}
& {\left[u_{1} \mid u_{1}=\right.\text { John }} \\
& \quad(\beta)\left[\mid \operatorname{has}-\operatorname{to}\left[\mid \operatorname{clean}\left(u_{1}\right)\right]\right] \Rightarrow\left[\mid \neg \operatorname{want}\left(u_{1}, \operatorname{clean}\left(u_{1}\right)\right)\right] \\
& \left.\quad(\alpha)\left[\mid \operatorname{has}-\operatorname{to}\left[\mid \operatorname{cook}\left(u_{1}\right)\right]\right] \Rightarrow\left[\mid \neg \operatorname{want}\left(u_{1}, \operatorname{cook}\left(u_{1}\right)\right)\right]\right]
\end{aligned}
$$


Using our generalization operations, we can construct the following maximal common theme MCT1 by $\lambda$-abstracting over $C$ in both $\alpha$ and $\beta$ :

$$
\text { MCT1 } \lambda C\left[u 1 \mid u 1=J o h n\left[\mid \text { has-to }\left[\mid C\left(u_{1}\right)\right]\right] \Rightarrow\left[\mid \neg \operatorname{want}\left(u_{1}, C\left(u_{1}\right)\right)\right]\right]
$$

Consider now the bad reading, shown in (29).

$$
\begin{aligned}
& {\left[u_{1} \mid u_{1}=\right.\text { John }} \\
& \quad(\beta)\left[\mid \text { has-to }\left[\mid \operatorname{clean}\left(u_{1}\right)\right]\right] \Rightarrow\left[\mid \neg \operatorname{want}\left(u_{1}, \operatorname{help}\left(u_{1}, m e\right)\right)\right] \\
& \left.\quad(\alpha)\left[\mid \operatorname{has}-\operatorname{to}\left[\mid \operatorname{cook}\left(u_{1}\right)\right]\right] \Rightarrow\left[\mid \neg \operatorname{want}\left(u_{1}, \operatorname{clean}\left(u_{1}\right)\right)\right]\right]
\end{aligned}
$$

The best we can do with the resolved ellipses in (29) is to abstract over the terms in the consequent, replacing them with the variable $C^{\prime}$ to get the theme MCT2.

$$
\text { MCT2 } \lambda C^{\prime} \lambda C\left[u 1=J o h n,\left[\mid \text { has-to }\left[\mid C\left(u_{1}\right)\right]\right] \Rightarrow\left[\mid \neg \text { want }\left(u_{1}, C^{\prime}\right)\right]\right]
$$

In MCT2, there is $\lambda$-abstraction over the VPs in the antecedents and a separate abstraction over the consequents. Since an additional generalization operation is required to produce MCT2, there is a preference for MCT1, which accounts for the preference for the good sloppy reading of (22). According to Asher 1993; Asher et al. 2001, the PARALLEL relation requires that the MCT have a minimal amount of content. The theme MCT2 for (29) has almost no content whatsoever, and so the account would predict that this reading is not only not preferred but it is degraded as well.

We have shown how our approach rules out bad sloppy readings for (22) by relying on discourse relations and the computation of MCT. Furthermore, we note that there is a preference for the reading in which both VPE occurrences are resolved to help me with my writing. In our view, the first sentence introduces a subject for the overall discussion and so makes the above strict reading most coherent. One can imagine other interpretations which bring to the fore the sloppy reading (clean/cook) or the other strict reading (clean/clean). However, our contention is that the bad sloppy readings are systematically dispreferred or ruled out.

We finish up this section by returning to (10). The two resolutions of the ellipses and underspecified terms introduced rely on two different MCTs. The first one is:

$$
\begin{aligned}
& -\lambda C\left[u_{1}, \mid u_{1}=J o h n,\right. \\
& \quad\left[u_{2} \mid \operatorname{teacher}\left(u_{2}\right), \operatorname{insists}\left(u_{2}, \wedge\left[\mid\left[u_{3} \mid \operatorname{student}\left(u_{3}\right)\right] \Rightarrow \operatorname{has}-\operatorname{to}\left[\mid C\left(u_{3}, u_{2}\right)\right]\right]\right)\right] \\
& \left.\quad \Rightarrow\left[\mid \neg \operatorname{want}\left(u_{1}, C\left(u_{1}, u_{2}\right)\right)\right]\right]
\end{aligned}
$$

This theme is associated with the sloppy reading of (10) represented in (19) and (20). It was generated by abstracting over both the overt VP (show her respect) and the elided VP with the same content.

The second MCT is generated by abstracting only over the overt VP: 
VP ellipsis without indices

- $\lambda C\left[u_{1}, \mid u_{1}=J o h n\right.$,

$\left[u_{2} \mid \operatorname{teacher}\left(u_{2}\right), \operatorname{insists}\left(u_{2}, \wedge\left[\mid\left[u_{3} \mid \operatorname{student}\left(u_{3}\right)\right] \Rightarrow\right.\right.\right.$ has-to $\left.\left.\left.\left[\mid C\left(u_{3}, u_{2}\right)\right]\right]\right)\right]$

$\Rightarrow\left[\mid \neg \operatorname{want}\left(u_{1}, \operatorname{show}\left(u_{1}, u_{2}\right.\right.\right.$, respect $\left.\left.\left.)\right)\right]\right]$

The second theme yields the following strict reading of the ellipsis in the second sentence:

$$
\begin{aligned}
& {\left[u_{1}, \mid u_{1}=J \text { ohn },\right.} \\
& {\left[u_{2} \mid \operatorname{teacher}\left(u_{2}\right),\right.} \\
& \left.\left.\left.\quad \text { insists }\left(u_{2}, \wedge|| u_{3} \mid \operatorname{student}\left(u_{3}\right)\right] \Rightarrow \text { has-to }\left[\mid \operatorname{show}\left(u_{3}, u_{2}, \text { respect }\right)\right]\right]\right)\right] \\
& \quad \Rightarrow\left[\mid \neg \operatorname{want}\left(u_{1}, \operatorname{show}\left(u_{1}, u_{2}, \operatorname{respect}\right)\right)\right] \\
& {\left[u_{4} \mid \operatorname{teacher}\left(u_{4}\right),\right.} \\
& \left.\quad \operatorname{says}\left(u_{4}, \wedge\left[\mid\left[u_{5} \mid \operatorname{student}\left(u_{5}\right)\right] \Rightarrow \text { has-to }\left[\mid \operatorname{give}\left(u_{5}, u_{4}, \operatorname{presents}\right)\right]\right]\right)\right] \\
& \left.\quad \Rightarrow\left[\mid \neg \operatorname{want}\left(u_{1}, \operatorname{show}\left(u_{1}, u_{4}, \operatorname{respect}\right)\right)\right]\right]
\end{aligned}
$$

Note that neither MCT can be derived from the other by means of our generalizing operations, and so neither is more maximal than the other. From this we predict that the two readings associated with these logical forms are both felicitous.

\subsubsection{Other discourse relations and VPE}

The requirement for parallel controllers that we have been discussing does not arise from ellipsis or anaphora, but rather from the discourse relations PARALLEL and CONTRAST. ${ }^{5}$ Other discourse relations are equally relevant. The sentences in (32) are related by INSTANCE, which does not require parallel controllers but nevertheless imposes a constraint that affects the resolution of ellipsis (example 32 is based on an example in Hardt 1993).

(32) Every boy in the class wants Ms. Jones to like him. In John's case, I'm sure she will.

$$
\begin{aligned}
& {\left[u_{1}, u_{2}, C_{1} \mid u_{1}=\text { Ms. Jones, } u_{2}=\operatorname{John}, C_{1}=\lambda u[z \mid \operatorname{like}(u, z), z=?]\right.} \\
& \quad(\alpha)\left[u_{3} \mid \operatorname{student}\left(u_{3}\right)\right] \Rightarrow\left[\mid \operatorname{want}\left(u_{3}, C_{1}\left(u_{1}\right)\right)\right] \\
& \left.\quad(\beta)\left[\mid \operatorname{in} \operatorname{case}\left(u_{2}\right), \operatorname{sure}\left(i, \wedge\left[C_{2} \mid \operatorname{will}\left(C_{2}\left(u_{1}\right)\right)\right]\right), C_{2}=?\right]\right]
\end{aligned}
$$

The following definition, based on Asher 1993, provides the relevant constraint.

- INSTANCE $(\alpha, \beta)$ iff there is a map $\mu$ from $\beta$ to $\alpha$ such that: (i) for at least one individual term $t$ in $\beta, \mu(t)$ is a quantifier in $\alpha$; (ii) for any modal operator $m$ in $\beta \mu(m)$ is a modal operator in $\alpha$; and (iii) abstracting over all elements paired in $\mu$ yields a common theme.

5 We did not explicitly discuss CONTRAST, but CONTRAST requires contrasting controllers, which yield two conflicting themes (Asher 1993; Asher et al. 2001) under the operations of generalization. 
INSTANCE requires the DRS $\beta$ to supply a witness for the nuclear scope of $\alpha$; in the case of (32), $z=$ ? in the description of $C_{1}$ must be resolved to $z=u_{2}$. Thus while there is no parallel controller requirement for (32), we see that other discourse relations can lead to particular preferences for the resolution of anaphoric elements.

(34) shows a rather different way in which ellipsis resolution is governed by discourse relations.

John said that Sam flunked the exam.

a. No he didn't.

b. But he didn't.

(34a) is an example of CORRECTION whereas (34b) is a more familiar example of CONTRAST. The ellipsis in (34a) resolves to say that Sam flunked the exam while the ellipsis in (34b) resolves to flunked the exam. This is because CONTRAST is a veridical relation in the sense that it entails the truth of both of its terms, so the only possible consistent resolution of the anaphor in (34b) is to the lower VP. On the other hand, CORRECTION necessarily entails the falsehood of its first term, so the ellipsis in (34b) is naturally resolved to the higher VP.

Relations that indicate causality, such as EXPLANATION and RESULT, exercise constraints on VPE as well, as shown by the following minimal pair:

(35) Someone has to clean this house. Although Sam doesn't want to, I'm going to hire a maid.

(36) Someone has to clean this house. Because Sam doesn't want to, I'm going to hire a maid.

Whenever there is an underspecified element in a clause that is an argument for a discourse relation, this element must be resolved to a contextual value that maximizes the plausibility of the relation indicated (Asher \& Lascarides 2003). In (35), although is a non-ambiguous marker for CONTRAST so we know that although Sam doesn't want to is related to I'm going to hire a maid by CONTRAST and the VPE must be resolved so as to maximize the strength of the contrast. If we resolve the VPE to clean this house, we get a very weak and even incoherent CONTRAST relation with not wanting to clean the house and hiring a maid as the two themes (where one theme should defeasibly entail or raise the probability of the negation of the other). On the other hand, by resolving the VPE to hire a maid, we achieve a much stronger CONTRAST with not wanting to hire a maid and hiring a maid as the two contrasting themes. In (36), however, the relevant clauses are related by EXPLANATION, as signalled by the non-ambiguous EXPLANATION marker, because. In this case, maximizing the plausibility of the discourse relation leads to different resolution 
VP ellipsis without indices

preferences for the VPE. Resolving to clean the house is far more felicitous than in (35) - and even preferred-because it provides a natural explanation for why the speaker is going to hire a maid.

The behavior of ellipsis with regard to constraints imposed by discourse structure echoes the behavior of other anaphoric elements. ${ }^{6}$ Recent work (Kehler, Kertz, Rohde \& Elman 2008) on the resolution of anaphoric elements has shown finegrained differences in resolution strategies depending on the discourse relations operative in the context.

\section{Conclusions}

In this paper we have addressed the following question: should indices be introduced at the syntactic level or should they rather be treated as the output of a semantic and pragmatic process? We have examined data involving sloppy VPE, in which, we claim, ellipsis sites are to be represented as variables. We have shown that this data presents fundamental problems for a structural view of indices, both because of problems concerning accessibility, and because of the problem of parallel controllers. Our solution has two parts. First, we reject pre-indexed logical forms, opting instead for underspecified representations, in which indices represent the result of a resolution process. Second, we show how discourse relations constrain this resolution process.

\section{References}

Asher, Nicholas. 1993. Reference to Abstract Objects in Discourse. Dordrecht: Kluwer.

Asher, Nicholas, Daniel Hardt \& Joan Busquets. 2001. Discourse parallelism, ellipsis, and ambiguity. Journal of Semantics 18(1). 1-25.

Asher, Nicholas \& Alex Lascarides. 2003. Logics of Conversation. Cambridge: Cambridge University Press.

Charlow, Simon. 2012. Cross-categorial donkeys. In Maria Aloni \& Frank Veltman (eds.), Eighteenth Amsterdam Colloquium, 171-192. Amsterdam: Foris.

Dalrymple, Mary, Stuart M. Sheiber \& Fernando C. N. Pereira. 1991. Ellipsis and higher-order unification. Linguistics and Philosophy 14(4). 399-452.

Fiengo, Robert \& Robert May. 1994. Indices and Identity. Cambridge, MA: MIT Press.

de Groote, Philippe. 2006. Towards a Montegovian account of dynamics. In Semantics and Linguistic Theory (SALT) 16, 148-155. CLC Publications.

6 Asher (1993) argues in detail for a parallel between VPE and pronouns referring to abstract entities. 
Hardt, Daniel. 1993. Verb phrase ellipsis: Form, meaning, and processing: University of Pennsylvania dissertation. Distributed as IRCS Report 93-23.

Hardt, Daniel. 1994. Sense and reference in dynamic semantics. In Paul Dekker \& Martin Stokhof (eds.), Ninth Amsterdam Colloquium, 333-348. Amsterdam: Foris.

Hardt, Daniel. 1999. Dynamic interpretation of VP ellipsis. Linguistics and Philosophy 22(2). 187-221.

Kehler, Andrew, Laura Kertz, Hannah Rohde \& Jeffrey Elman. 2008. Coherence and coreference revisited. Journal of Semantics (Special Issue on Processing Meaning) 25(1). 1-44.

Merchant, Jason. 2012. Ellipsis. To appear in Handbook of Contemporary Syntax, 2nd edition, Tibor Kiss and Artemis Alexiadou eds., De Gruyter, Berlin.

Muskens, Reinhard. 1996. Combining Montague semantics and discourse representation. Linguistics and Philosophy 19(2). 143-186.

Prust, Hub, Remko Scha \& Martin van den Berg. 1994. A discourse perspective on verb phrase anaphora. Linguistics and Philosophy 17(3). 261-327.

Rooth, Mats Edward. 1992. A theory of Focus interpretation. Natural Language Semantics 1(1). 75-116.

Sag, Ivan A. 1976. Deletion and logical form: MIT dissertation.

van der Sandt, Robert. 1992. Presupposition projection as anaphora resolution. Journal of Semantics 9. 333-377.

Sauerland, Uli. 2007. Copying vs. Structure Sharing: A Semantic Argument. In Linguistic variation yearbook 7, 27-51.

Schwartz, Bernhard. 2000. Topics in ellipsis: University of Massachusetts at Amherst dissertation.

Tomioka, Satoshi. 2008. A step-by-step guide to ellipsis resolution. In Kyle Johnson (ed.), Topics in ellipsis, 210-228. Cambridge: Cambridge University Press.

Williams, Edwin. 1977. Discourse and logical form. Linguistic Inquiry 8(1). 101-139.

Daniel Hardt

IT Management

Copenhagen Business School

2000 Frederiksberg, Denmark

dh.itm@cbs.dk

Julie Hunter

Centre Universitaire Jean François Champollion Place Verdun

81012 Albi, France

juliehunter@gmail.com
Nicholas Asher

CNRS, IRIT UMR 5505

Université Paul Sabatier

31062 Toulouse, France

asher@irit.fr 